\title{
An S-shaped arc in the galaxy cluster RX J0054.0-2823 ${ }^{\star}$
}

\author{
C. Faure ${ }^{1,4}$, E. Giraud ${ }^{2}$, J. Melnick ${ }^{3}$, H. Quintana ${ }^{4}$, F. Selman ${ }^{3}$, and J. Wambsganss ${ }^{1}$ \\ 1 Zentrum für Astronomie der Universität Heidelberg (ZAH), Mönchhofstr. 12-14, 69120 Heidelberg, Germany \\ 2 Laboratoire Physique Théorique et Astroparticules, UMR5207 In2p3/Montpellier University, 34095 Montpellier, France \\ e-mail: edmond.giraud@lpta.in2p3.fr \\ 3 European Southern Observatory, Alonso de Córdova 3107, Santiago, Chile \\ 4 Department of Astronomy and Astrophysics, P. Universidad Catolica de Chile, Casilla 306, Santiago, Chile \\ Received 19 February 2006 / Accepted 29 September 2006
}

\begin{abstract}
Context. The center of the galaxy cluster RX J0054.0-2823 at $z=0.292$ is a dynamically active region that includes an interacting system of three galaxies surrounded by a large halo of intra-cluster light.

Aims. We report here the discovery of an S-shaped feature of total length $11^{\prime \prime}$ in the central region of this cluster and discuss its physical nature.

Methods. We test the gravitational lensing assumption by doing a mass modeling of the central part of the galaxy cluster.

Results. We very naturally reproduce position and form of this S-shaped feature as a gravitationally lensed background object at redshift between 0.5 and 1.0 .

Conclusions. We conclude that the lensing nature is the very probable explanation for this S-shaped arc; the ultimate proof will be the spectroscopic confirmation by measuring the high redshift of this elongated feature with surface brightness $V \sim 24 \mathrm{mag}^{\operatorname{arcsec}}{ }^{-2}$.
\end{abstract}

Key words. cosmology: observations - galaxies: clusters: individual: RX J0054.0-2823 - galaxies: interactions - galaxies: halos gravitational lensing

\section{Introduction}

Giant luminous arcs were discovered in 1986/87 (Lynds \& Petrosian 1986; Soucail et al. 1987) and found to be highly distorted background galaxies, magnified and often multiply imaged by the gravitational lensing action of an intervening galaxy cluster (Paczyński 1987). Meanwhile, more than 100 giant arcs systems have been found (Gladders et al. 2002, and references therein) and studied both to infer the cluster mass distribution and - using the magnification effect - to investigate the high redshift galaxy population (e.g., Colley et al. 1996). The ensemble of arcs was also used for statistical inferences regarding consistency with various cosmological models (e.g., Bartelmann et al. 1998; Golse et al. 2002; Soucail et al. 2004; Wambsganss et al. 2004; Sand et al. 2005).

Contrary to the implication of the name, the shapes of highly distorted background galaxies are not always curved like an arc: lots of radial arcs have been found with elongated features extended in the radial direction; a straight arc was discovered early on as well (Wambsganss et al. 1989). Here we report the discovery of a strangely shaped arc candidate. Our modeling shows that a very natural explanation for this $\mathrm{S}$-shaped feature is a background galaxy gravitationally lensed by the moderately massive X-ray cluster RX J0054.0-2823, with the shape particularly affected by the three prominent central galaxies and the cluster ellipticity.

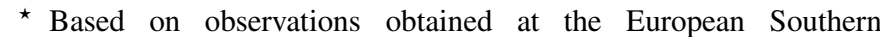
Observatory, La Silla, Chile and ESO archive files.

\section{Observations and data reduction}

The galaxy cluster RX J0054.0-2823 was identified as part of the 160 square degree ROSAT serendipity survey (Vikhlinin et al. 1998; Mullis et al. 2003). Our imaging observations of RX J0054.0-2823 consist of a series of $24 \mathrm{~V}$-band and 39 I-band images, with an exposure time of $300 \mathrm{~s}$ each, acquired during two photometric dark nights on September 26 and 27, 2000, using SUSI 2 at the ESO NTT. The total exposure time was $18900 \mathrm{~s}$. SUSI 2 covers a field of $5^{\prime} \times 5^{\prime}$ with two CCDs at a pixel scale of $0.0805^{\prime \prime}$ pixel $^{-1}$. The CCDs were binned $2 \times 2$. To correct for fringing, sky background, and CCD variations, the observations were carried out with an alternating dithering pattern as described in Melnick et al. (1999). In this method, the cluster center is placed on one of the CCDs, say CCD1, while CCD2 simultaneously measures the sky background. Then the cluster is centered on CCD2, and CCD1 is used to measure the sky. The exposure is thus divided in a large number of integrations, nodding consecutively on CCD1 and CCD2, and offsetting the telescope by small random amounts to dither the images. The differences between consecutive images allow us to correct for fringing, to build and subtract for darks, and to remove most systematic effects. The frames are flat-fielded by using a master flat. The final images, which are the medians of individual 300 s exposures, are calibrated using Landolt stars (1992). A maximum variation by a factor of 2 was found in the $I$-band sky background over the two nights, with 3 main groups of values at 19.2, 19.5, and $19.9 \mathrm{mag} \operatorname{arcsec}^{-2}$. The background noise depends on the number of individual images at a given position: in the central overlap area, the $1 \sigma$ isophotes are at $V=27.0$ and $I=25.7 \mathrm{mag} \operatorname{arcsec}^{-2}$, respectively. The connection between 


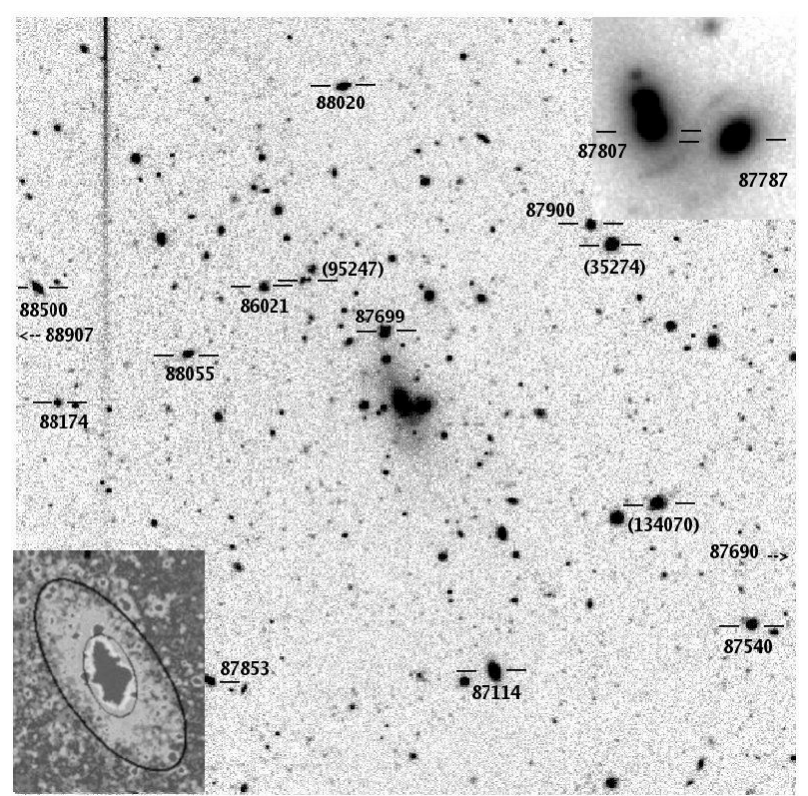

Fig. 1. The $V$-band image $\left(4^{\prime \prime} \times 4^{\prime \prime}\right)$ in log intensity scale centered on RX J0054.0-2823. The total exposure time is $7200 \mathrm{~s}$, and the seeing $0.8^{\prime \prime} F W H M$. The radial velocities are super-imposed on the galaxies (in $\mathrm{km} \mathrm{s}^{-1}$ ). Velocities of galaxies outside the field are marked by arrows. The velocity $95247 \mathrm{~km} \mathrm{~s}^{-1}$ corresponds to two galaxies. Therefore, we have measured the velocity dispersion for 18 objects in the field. The upper insert is the central $16^{\prime \prime} \times 16^{\prime \prime}$ region where the S-shaped feature is visible between the three central galaxies. The lower insert is the $59^{\prime \prime} \times 75^{\prime \prime}$ central region binned to $9 \times 9$ pixels, and it shows the intergalactic light. The inner (outer) ellipse delineates the $V=27(31) \mathrm{mag} \operatorname{arcsec}^{-2}$-contour. Both have similar ellipticities $\epsilon=1-b / a \sim 0.4$ and orientations, $\theta \sim 20^{\circ}\left(\sim 30^{\circ}\right)$. North is to the top, East is to the left.

pixels of real objects allows clear detections at these magnitudes. The full field size of our final image is $9.5^{\prime} \times 5.5^{\prime}$.

The galaxy spectra of RX J0054.0-2823, which we retrieved from the ESO archives (program 64.O-0455(A); P.I. H. Quintana), were originally obtained using EFOSC in multiobject spectroscopic mode (MOS) at the ESO $3.6 \mathrm{~m}$ telescope. Two consecutive MOS spectra of the same galaxies with $1800 \mathrm{~s}$ exposure time each had been acquired during the night of November 8, 1999, with atmospheric seeing of $0.9^{\prime \prime}$ and $0.8^{\prime \prime}$, respectively. They had been obtained with slitlets of $1.5^{\prime \prime}$ and Grism \#6, which provides a wavelength coverage of $380-850 \mathrm{~nm}$ at a resolution of $2 \mathrm{~nm}(R \sim 300)$. The detector was binned $2 \times 2$ at readout, giving a spectral dispersion of $0.4 \mathrm{~nm} \mathrm{pixel}^{-1}$ and a spatial scale of $0.31^{\prime \prime}$ pixel $^{-1}$. The data were analyzed using the context LONG within MIDAS. The two-dimensional spectra were corrected for bias, flat-field, and wavelength calibrated using HeAr lines. One-dimensional spectra were then extracted after sky subtraction. Redshifts were measured by fitting the continuum and the identified lines by using the MIDAS context ALICE. They are based on [OII], the $\mathrm{CaII} \mathrm{H}$ and $\mathrm{K}$ doublet, the Mgb band, NaID, and the Balmer lines. Eighteen individual galaxy redshifts were thus obtained (all indicated in Fig. 1), out of which 14 turned out to be cluster members (see also redshift histogram in Fig. 3).

\section{The galaxy cluster: images and redshifts}

The $V$-band image of galaxy cluster RX J0054.0-2823 is shown in Fig. 1. On a $4^{\prime}\left(1.3 h_{75}^{-1} \mathrm{Mpc}\right)$ scale, the main feature is an apparently elongated distribution of galaxy traced by a few bright galaxies at $\mathrm{PA} \sim 30^{\circ}$ with a concentration around 3 major objects in close contact, surrounded by a common envelope, which is also the location of the X-ray emission detected by ROSAT (Mullis et al. 2003). The central galaxy triplet (see top right insert of Fig. 1) is made up of a close pair of elliptical galaxies (labeled \#131 and \#139 in Fig. 4) and a more elongated object (\#128). On a scale of $25^{\prime \prime}\left(\sim 0.14 h_{75}^{-1} \mathrm{Mpc}\right)$, our deep images show that the central objects are surrounded by extended intra-cluster light elongated in the direction of the distribution of the galaxies described above (cf. Fig. 1, bottom left insert). The size of this intra-cluster light component at the $V=27 \mathrm{mag} \operatorname{arcsec}^{-2}$ isophote is $\sim 30^{\prime \prime} \times 16.7^{\prime \prime}$, and 2 to 3 times larger at the $V=31 \mathrm{mag} \operatorname{arcsec}^{-2}$ isophote. There are several very faint clumps in this region surrounded by the diffuse intra-cluster light. The ROSAT X-ray map is too noisy to measure an elongation, but its core radius is about $r_{\text {core }}=37 \pm 6^{\prime \prime}$ (or $200 \pm 32 h_{75}^{-1} \mathrm{kpc}$ ) from Vikhlinin et al. (1998). With redshifts for only 14 galaxies and with a limited spatial coverage, it is difficult to estimate the mass of the cluster. We use the standard virial mass estimator (Limber \& Mathews 1960; Carlberg et al. 1996), but we complement our estimates with an independent check from the measured X-ray luminosity for this cluster:

$M_{\mathrm{v}}=\frac{3}{G} \sigma_{1 \mathrm{D}}^{2} r_{\mathrm{v}}$

where $\sigma_{1 \mathrm{D}}$ is the line-of-sight velocity dispersion, and $r_{\mathrm{V}}$ is the virial radius defined as $r_{\mathrm{v}}=\pi R_{\mathrm{h}} / 2$, with $R_{\mathrm{h}}$ the projected harmonic radius

$R_{\mathrm{h}}=\left\langle\frac{1}{\left\|\boldsymbol{R}_{i}-\boldsymbol{R}_{j}\right\|}\right\rangle_{\text {Pairs }}$,

where $\boldsymbol{R}_{i}$ is the projected galaxy position vector. The galaxies used to perform this analysis are chosen to be at a distance equal to or lower than $0.25 \mathrm{mag}$ to the red sequence at redshift $z=0.25$. This selection criteria is illustrated in Fig. 2. The above estimates are valid only when the distribution of galaxies is spherically symmetric, and in our case we note a considerable elongation in the density distribution. We do only an order or magnitude estimate in this section, pointing out that it has been noticed in the past that spherical dynamic models can accurately reproduce the dynamics of slightly elongated systems (Sand et al. 2002; Kronowitter et al. 2000).

The redshift distribution of the 18 objects is shown in Fig. 3: 14 of the 18 measured galaxies are cluster members with velocities around $\sim 88000 \mathrm{~km} \mathrm{~s}^{-1}$. The remaining four galaxies are a very close pair of faint objects at $\sim 95250 \mathrm{~km} \mathrm{~s}^{-1}$, a background galaxy at $134070 \mathrm{~km} \mathrm{~s}^{-1}$, and a foreground galaxy at $35275 \mathrm{~km} \mathrm{~s}^{-1}$ (not plotted in the histogram). For the cluster members, the distribution is well peaked at $c z=$ $87775 \mathrm{~km} \mathrm{~s}^{-1}$, with a dispersion $\sigma=675 \mathrm{~km} \mathrm{~s}^{-1}$. The average coincides with the mean of two of the main central galaxies (\#128 and \#131), in good agreement with the redshift $z=0.292$ given by Mullis et al. (2003) for the cluster. The internal error in the galaxy redshifts is $\Delta v=209 \pm 30 \mathrm{~km} \mathrm{~s}^{-1}$, so the intrinsic line-of-sight (rest frame) radial velocity dispersion of the cluster is $\sigma_{1 \mathrm{D}}=\sqrt{\sigma^{2}-\Delta v^{2}} /(1+z)=497 \mathrm{~km} \mathrm{~s}^{-1}$, and the $3 \mathrm{D}$ dispersion $\sigma_{3 \mathrm{D}}=\sqrt{3} \sigma_{1 \mathrm{D}}=860 \mathrm{~km} \mathrm{~s}^{-1}$. The harmonic radii of the galaxies with velocities and of the galaxies in the red sequence are $410 h_{75}^{-1} \mathrm{kpc}^{-1}$ and $710 h_{75}^{-1} \mathrm{kpc}^{-1}$, respectively. With this value for the velocity dispersion, and with the above values for the harmonic radius, we obtain a virial 

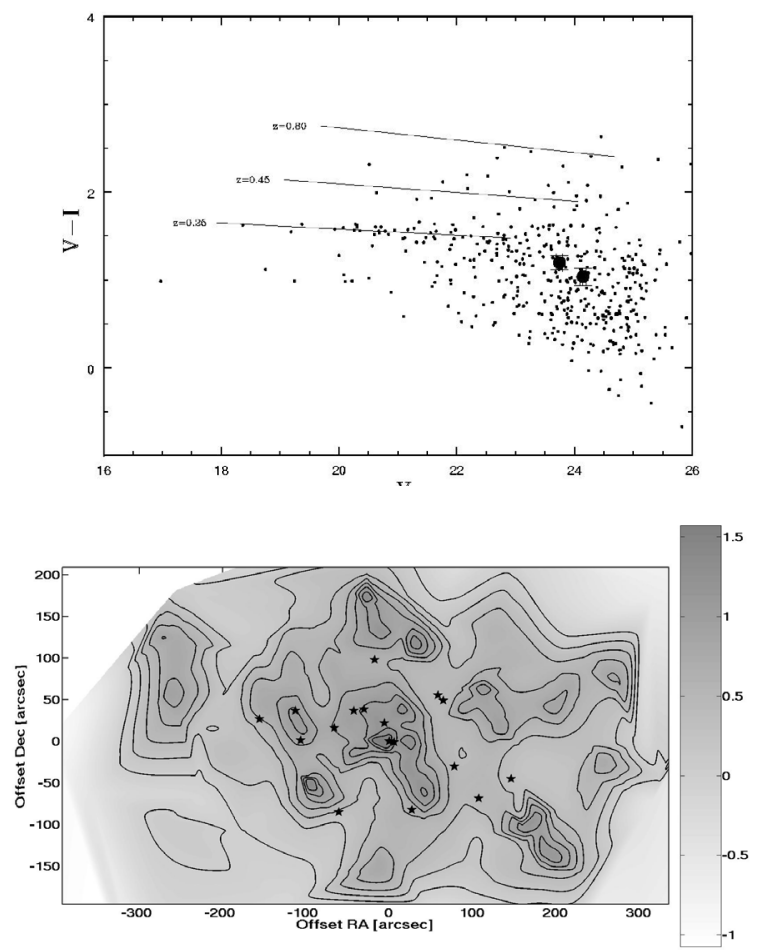

Fig. 2. Top panel: cluster color-magnitude diagram showing the positions of the red sequence, the two arcs (big black dots), and the locus of red sequence expected for redshifts of 0.25, 0.45, and 0.80 according to Kodama et al. (1998). Bottom panel: contour map of the $\log _{10}$ of galaxy density selecting only galaxies within 0.25 mag from the red sequence. The stars mark the position of galaxies with measured redshift. The density is in unit of galaxies per square arcminute, and the contours are separated by 0.2 dex intervals. North is to the top, East is to the left.

cluster mass in the range $(1-2) \times 10^{14} h_{75}^{-1} M_{\odot}$. The luminosity of the galaxies in the red sequence within the corresponding radii are $1.76 \times 10^{11} h_{75}^{-2} L_{V \odot}$ and $3.06 \times 10^{11} h_{75}^{-2} L_{V \odot}$. The derived $M / L$ ratios are $570 h_{75} M_{\odot} / L_{V \odot}$, and $650 h_{75} M_{\odot} / L_{V \odot}$, respectively. These large $M / L$ values must be considered upper limits since they are derived considering only the galaxy part of the red sequence. The X-ray luminosity of the cluster is $L_{\mathrm{X}}=0.63 \times 10^{44} h_{75}^{-2} \mathrm{erg} \mathrm{s}^{-1}$ (Vikhlinin et al. 1998). According to Reiprich \& Böringer (2002), with this luminosity and the mass determined above, RX J0054.0-2823 is right in the correlation between X-ray luminosity and cluster virial mass in the region of the least massive clusters.

The approximate linear arrangement of the galaxies, and the clumpy general appearance suggest that the region is probably dynamically young and that the triple central galaxy system could be the merging progenitor of a future single large cD. In addition to the X-ray emitting hot gas, the central potential well appears to be filled with smooth intra-cluster stellar light and the faint sub-galaxian clumps could be material ejected from the interacting system. A composite spectrum in the wavelength range $365-420 \mathrm{~nm}$ was built with the 14 cluster galaxy spectra to characterize the presence of star formation following the method of Dressler et al. (2004). This spectrum indicates that there has been no significant star formation in this region for several Gyr (e.g., weak $\mathrm{H} \delta$ and $[\mathrm{OII}])$.

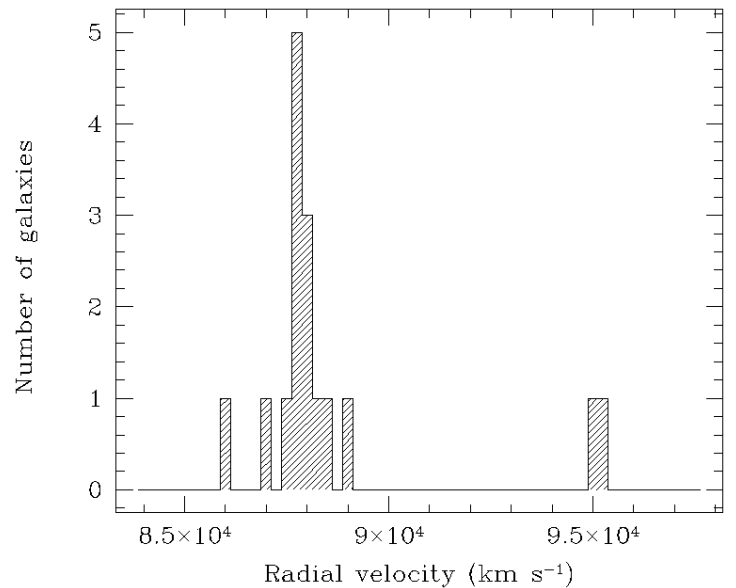

Fig. 3. Velocity histogram of the 16 galaxies in the central region of RX J0054.0-2823 (bin size $250 \mathrm{~km} \mathrm{~s}^{-1}$; two galaxies outside range).

\section{The S-shaped arc}

As shown in Fig. 1 (insert top right) and in Fig. 4 (top left panel), there is a giant arc with a total length of $\sim 11^{\prime \prime}$ and an unresolved width $\left(\leq 0.70^{\prime \prime}\right)$ superimposed on the intra-cluster light. The arc-like feature has the shape of an "S", slightly inclined South-East to North-West. It embraces the South and East part of galaxy \#131 and the West and North part of galaxy \#128. The bright northern and southern extensions of the arc have a surface brightness of $V \sim 24 \mathrm{mag} \operatorname{arcsec}^{-2}$, while there is a drop in luminosity in the region that links them.

The $(V-I)$-color of the arc is $\sim 1.1 \pm 0.1$ for the North component and $\sim 1.0 \pm 0.1$ for the fainter South component (see Fig. 2, top panel). There are various possibilities for the physical origin of this S-shaped arc. The color of the arc is consistent with being late-type gravitationally lensed galaxies at high redshift. But it is also consistent with being cluster member(s) in the faint end of the luminosity function that are being tidally deformed in an interaction with the central multiple system. In that case, this S-shaped feature could be either tidal debris from the merging of the massive central galaxies, or one or two tidally disrupted cluster members. At $V \sim 24 \mathrm{mag} \operatorname{arcsec}^{-2}$ it would be much brighter than the tidal features found in Virgo (e.g., Mihos et al. 2005), especially if we consider the Tolman dimming, which at this redshift should be approximately 1 mag. Thus, although possible, we consider the tidal debris origin to be unlikely. A tidally disrupted galaxy interacting with the bright central galaxies is a possibility that cannot be dismissed. A disk galaxy could in principle create a long tidal tail in the interaction with one of these galaxies, which could then be distorted by the other. Although this scenario is highly unlikely a priori, we must remember that it was precisely the peculiar geometry of the system that caught our attention in the first place. In what follows we explore the gravitational lens scenario by modeling the arc-shape with the known galaxy and cluster potentials.

\subsection{Gravitational lens modelling}

To model the S-shaped arc as one or more gravitationally lensed background galaxies, we need to know the gravitational potentials of the putative deflectors. These can be estimated using the structural parameters of the galaxies as given by their photometry and velocity dispersions. The latter have been estimated from the $\mathrm{H}$ and $\mathrm{K}$ line-profile widths corrected for instrumental broadening by means of the (weak) emission lines. 


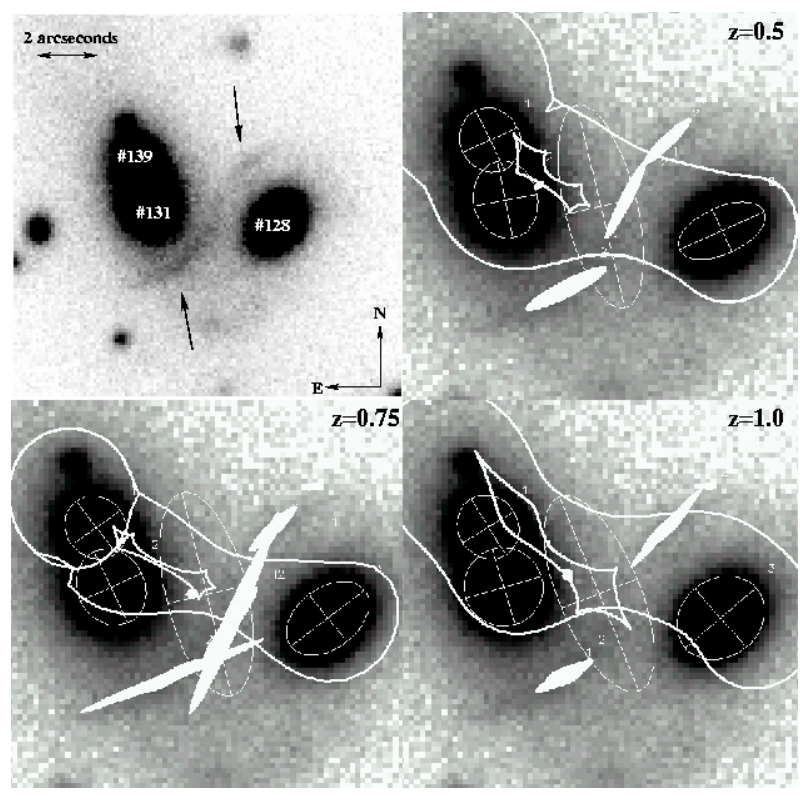

Fig. 4. Top left panel: the S-shaped feature in the $10^{\prime \prime} \times 10^{\prime \prime} V$-band image of the center of RX J0054.0-2823. The names of the galaxies are labeled as in the text and in Table 1. The arrows point at the bright regions of the arc. Top right panel and bottom panels: the lens model for three different source redshifts (indicated in the upper-right corner of each image) superimposed on the central $6^{\prime \prime} \times 6^{\prime \prime} V$-band image. North is to the top, East is to the left. The ellipsoids filled in white show the location of the images (the source position is represented by a white dot) for the best lens mass model. The white ellipsoids indicate the location, ellipticity, and orientation of the lensing galaxies (\#128, \#131, and \#139) and of the galaxy cluster, as given in Table 1. For the galaxy cluster the size is reduced to optimize its visibility in the figure. The white bold solid lines indicate the inner and outer caustic lines for the best lens mass model.

The velocity dispersions turn out to be similar $\sim 370 \mathrm{~km} \mathrm{~s}^{-1}$ for the two main galaxies \#128 and \#131, corresponding to masses $M_{24}=(3.5-3.8) \times 10^{11} h_{75}^{-1} M_{\odot}$ and $M / L \operatorname{ratios}(M / L)_{24} \approx$ $22 h_{75}^{-1}(M / L)_{\odot}$, measured at $V=24 \mathrm{mag} \operatorname{arcsec}^{-2}$. No spectra were taken for galaxy \#139, hence no velocity dispersion is measured.

We want to test whether the S-shaped arc can be the distorted, potentially multiple, image of one or more background galaxies. Our simplified lens potential consists of four components at $z=0.292$ : the cluster, and the three central galaxies: $\# 128$, \#131, and \#139 (see Fig. 4). These components are fitted by Pseudo-Isothermal Mass Distributions (PIEMD, Kassiola \& Kovner 1993; Kneib et al. 1996) defined by position, core radius, cut radius, orientation, ellipticity, and velocity dispersion. Since the source redshift is not known, we have computed models for four different source redshifts: $z_{\mathrm{s}}=0.5,0.75,1.0$, and 2.0.

Since the arc has two bright regions (see Fig. 4, top left panel), we initially assumed that it is built by the gravitationally distorted images of two background objects. With these assumptions we used the LensTool package (Kneib et al. 1993) to model the arc in an iterative way, varying the input parameters within the ranges allowed by the observations. For the cluster potential the only initial constraint was the velocity dispersion, but since the models are relatively insensitive (within reasonable values) to the cluster center position, we chose to keep it fixed at a value close to the centroid of the three main galaxies (cf. Fig. 4 and Table 1). We iterated until we found solutions that satisfied the following criteria: (a) input parameters consistent
Table 1. Parameters of the gravitational lens model.

\begin{tabular}{llccccc}
\hline \hline Source & Lens & $\Delta x\left({ }^{\prime \prime}\right)$ & $\Delta y\left({ }^{\prime \prime}\right)$ & $\epsilon$ & $\theta\left(^{\circ}\right)$ & $\sigma_{v}\left(\mathrm{~km} \mathrm{~s}^{-1}\right)$ \\
\hline$z_{\mathrm{s}}=0.5$ & $\# 128$ & -0.81 & -1.37 & 0.49 & -65 & 350 \\
& $\# 131$ & 2.43 & -0.89 & 0.20 & 11 & 401 \\
& $\# 139$ & 2.66 & 0.02 & 0.10 & 30 & 252 \\
& Cluster & 1.0 & -1.0 & 0.66 & 13 & 940 \\
\hline$z_{\mathrm{s}}=0.75$ & $\# 128$ & -0.81 & -1.37 & 0.34 & -51 & 354 \\
& $\# 131$ & 2.43 & -0.89 & 0.16 & 36 & 360 \\
& $\# 139$ & 2.66 & 0.02 & 0.10 & 25 & 217 \\
& Cluster & 1.0 & -1.0 & 0.62 & 13 & 811 \\
\hline$z_{\mathrm{s}}=1.0$ & $\# 128$ & -0.81 & -1.37 & 0.10 & -43 & 230 \\
& $\# 131$ & 2.43 & -0.89 & 0.02 & 30 & 150 \\
& $\# 139$ & 2.66 & 0.02 & 0.10 & 34 & 285 \\
& Cluster & 1.0 & -1.0 & 0.51 & 28 & 618 \\
\hline
\end{tabular}

with observations, and (b) lensed images match the morphology of S-arc. The code naturally converges to a single source for the two arc components; therefore, we supposed that the arc is formed by the distorted images of a single background source. For the fit of the best lens potential, we allow the 3D-velocity dispersion to vary in a $20 \%$ error-bar range around the value $870 \mathrm{~km} \mathrm{~s}^{-1}$ for the galaxy cluster and $370 \mathrm{~km} \mathrm{~s}^{-1}$ for galaxies \#128 and \#131. Galaxy \#139 being fainter than the two other galaxies of the model, we assume that its mass is lower and put an upper limit on its velocity dispersion of $300 \mathrm{~km} \mathrm{~s}^{-1}$.

\subsection{Results}

Our best-fit models for a gravitational lensing scenario are shown in Fig. 4 for three values of the source redshift $\left(z_{\mathrm{s}}=0.5\right.$, $0.75,1.0)$. A large sample of good fits can be found for the observed parameter error-bars. This illustrates the robustness of the gravitational lensing hypothesis and the high probability of having such an S-shaped arc formed in the heart of such a mass potential configuration.

The parameters of our best-fitting models are tabulated in Table 1 where for each source redshift we report the position relative to the center of the North bright component of the arc $(\Delta x$, $\Delta y$ ), the ellipticity (defined as $\epsilon=1-b / a$, where $a$ is the semimajor axis and $b$ is the semi-minor axis), position angle ( $\theta$, positive North to East counter-clockwise), and the velocity dispersion $\left(\sigma_{v}\right)$ of the lens components. Our best fits are obtained for source redshifts $0.5 \leq z_{\mathrm{s}}<1.0$. For $z=1$, we match the galaxy and cluster PA and ellipticity only for velocity dispersions much lower than the measured one (see Table 1). Moreover, we were not able to obtain good fits for $z_{\mathrm{s}} \geq 1$. In fact, the model parameters of the lens components depart increasingly from the observations as the source redshift increases.

According to the model for source redshift at $z_{\mathrm{s}}=0.5$ and 0.75 , the arc would be built by 3 images of a single background galaxy: two images in opposite orientation (mirror images) would build the northern part of the arc, one single image would be the southern part. The luminosity drop observed in the northern part of the arc would reflect a decreasing surface brightness region in the galaxy source, which would also be in the lens plane, the location where the two northern images partially overlap. We conclude that gravitational lensing is a natural and very likely explanation for the S-shaped arc observed in RX J0054.0-2823. 


\section{Summary and conclusion}

We report the discovery of an S-shaped arc in the very center of the galaxy cluster RX J0054.0-2823. We discuss various possibilities for the physical origin of the feature. Modeling the potential formed by three massive central galaxies plus the cluster itself, we obtained very good fits to the location and shape of this arc for a gravitationally lensed galaxy source at $0.5 \leq z_{\mathrm{s}}<1.0$. These models show that the $\mathrm{S}$-shaped feature is likely to be the triple gravitational image of a single background galaxy. There is not a unique satisfying solution for the modeling. This implies that lensing is a very natural explanation for the S-arc feature for the configuration observed for the galaxies and the galaxy cluster. All the best-fitting potentials of the cluster have an ellipticity and orientation consistent with the distribution of both the diffuse intra-cluster light, and of the X-ray emission. This, together with the elongated galaxy distribution, and the relatively large mass inferred from the Virial theorem, suggests that the cluster is dynamically young, and probably the result of a recent merging.

Given the success of the lensing modeling, the alternative explanations of tidally induced compression of the stellar intracluster medium, or a highly distorted cluster spiral appear rather unlikely. The gravitational lensing explanation only works if the redshift of the source is lower than $z_{\mathrm{s}} \sim 1$. Therefore, a straightforward test for the lensing hypothesis is to measure the redshift of the arc.

Acknowledgements. C.F. and J.W. are supported by the European Community's Sixth Framework Marie Curie Research Training Network Programme, Contract
No. MRTN-CT-2004-505183 "ANGLES" and granted by the ECOS-CONYCIT Commitee. H.Q. is grateful for partial support from the FONDAP Astrophysics Center.

\section{References}

Bartelmann, M., Huss, A., Colberg, J. M., Jenkins, A., \& Pearce, F. R. 1998, A\&A, 330, 1

Carlberg, R. G., Yee, H. K. C., Ellingson, E., et al. 1996, ApJ 462, 32

Colley, W. N., Tyson, J. A., \& Turner, E. L. 1996, ApJ, 461, L83

Dressler, A., Oemler, A. J., Poggianti, B. M., et al. 2004, ApJ, 617, 867

Gladders, M. D., Yee, H. K. C., \& Ellingson, E. 2002, AJ, 123, 1

Golse, G., Kneib, J.-P., \& Soucail, G. 2002, A\&A, 387, 788

Kassiola, A., \& Kovner, I. 1993, ApJ, 417, 450

Kneib, J.-P., Mellier, Y., Fort, B., \& Mathez, G. 1993, A\&A, 273, 367

Kneib, J.-P., Ellis, R. S., Smail, I., Couch, W. J., \& Sharples, R. M. 1996, ApJ, 471,643

Kronawitter, A., Saglia, R. P., Gerhard, O., \& Bender, R. 2000, A\&AS, 144, 53

Kodama, T., Arimoto, N., Barger, A., \& Aragón-Salamanca, A. 1998, A\&A, 334, 99

Landolt, A. U. 1992, AJ, 104, 340

Limber, D. N., \& Mathews, W. G. 1960, ApJ, 132, 286

Lynds, R., \& Petrosian, V. 1986, AAS, 18, 1014

Melnick, J., Selman, F., \& Quintana, H. 1999, PASP, 111, 1444

Mihos, J. C., Harding, P., Feldmeier, J., \& Morrison, H. 2005 ApJ, 631, 41

Mullis, C. R., McNamara, B. R., Quintana, H., et al. 2003, ApJ, 594, 154

Paczyński, B. 1987, Nature, 325, 572

Reiprich, T., \& Böringer, H. 2002, ApJ, 567, 716

Sand, D. J., Treu, T., \& Ellis, R. S. 2002, ApJ, L129

Sand, D. J., Treu, T., Ellis, R. S., \& Smith, G. P. 2005, ApJ, 627, 32

Soucail, G., Fort, B., Mellier, Y., \& Picat, J.-P. 1987, A\&A, 172, L14

Soucail, G., Kneib, J.-P., \& Golse, G. 2004, A\&A, 417, L33

Vikhlinin, A., McNamara, B. R., Forman, W., et al. 1998, ApJ, 502, 558

Wambsganss, J., Schneider, P., Giraud, E., \& Weiss, A. 1989, ApJ, 337, L73

Wambsganss, J., Bode, P., \& Ostriker, J. P. 2004, ApJ, 606, L93 